\title{
Durable low ice adhesion foams modulated by submicron pores
}

Tong Li, Yizhi Zhuo, Verner Håkonsen, Jianying He* and Zhiliang Zhang*

NTNU Nanomechanical Lab, Department of Structural Engineering, Norwegian University of Science and Technology (NTNU), 7491, Trondheim Norway.

\section{E-mail: jianying.he@,ntnu.no and zhiliang.zhang@ntnu.no}

\section{Apparent shear modulus of the coatings by nanoindentation tests.}

The unloading stiffness (S) of the coatings can be obtained from linear-fitting the slope of the initial part of the unloading curves, which are shown via the black fitting lines in Figure 5a. The reduced modulus of these coatings is calculated by the $E_{\mathrm{r}}=S / D$, where $D$ is the diameter of the cylindrical flat punch. ${ }^{1}$ Young's modulus of these samples is calculated by the equation: $1 / E_{\mathrm{r}}=\left(1-v^{2}\right) / E+\left(1-v_{\text {tip }}^{2}\right) / \mathrm{E}_{\text {tip }}$, where $v$ and $v_{\text {tip }}$ are Poisson's ratio of the material and diamond indenter, respectively, and $E$ and $E_{\text {tip }}$ are Young's modulus of the material and diamond indenter, respectively. Here, the value of $v$ for pristine Sylgard 184 coating was assumed to be equal to $0.5, v_{\text {tip }}=0.07$ and $E_{\text {tip }}=1140 \mathrm{GPa}$ (Young's modulus of the diamond indenter). ${ }^{1-2}$

The value of $v$ for porous foam were assumed as $v=\left(0.42 p^{2}-0.63 p+1\right) v_{\text {PDMS }}{ }^{3}$ where $p$ is the porosity of porous coatings, and $v_{\mathrm{PDMS}}$ is 0.5 . Because $E_{\mathrm{tip}}$ is much larger than $E$, the second part of this equation is negligible. Therefore, $E \approx E_{\mathrm{r}}\left(1-v^{2}\right)$. Meanwhile, the shear modulus can be calculated from the equation: $E=2 G(1+v)$, so the apparent shear modulus $G=0.5\left(1-v^{2}\right) /$ $(1+v) E_{\mathrm{r}} \cdot{ }^{3-4}$

\section{Mechanical properties of the bulk PDMS.}

The bulk PDMS samples were prepared by regulating the ratio of Sylgard 184 precursors. The bulk samples with variable weight ratio were fabricated for investigation. The obtained load- 
displacement curves are shown in Figure S9 a. Based on the above calculation method, the shear modulus of the bulk sample and corresponding fitted curve are shown in Figure S9 b. In particular, the sample prepared from weight ratio of 0.3 has a shear modulus of about $299 \mathrm{kPa}$.

\section{The smooth and porous surfaces of foams.}

The surface of $30 \%$ foam and the porous surface (prepared from Span 80 to Sylgard 184 kits with a ratio of 3:7) are used for comparison. As shown in Figure S6, the surface of 30\% coating is much smoother than the porous surface. When the Tween 80 is introduced into the porogen system, the surface of the coatings become smoother. 

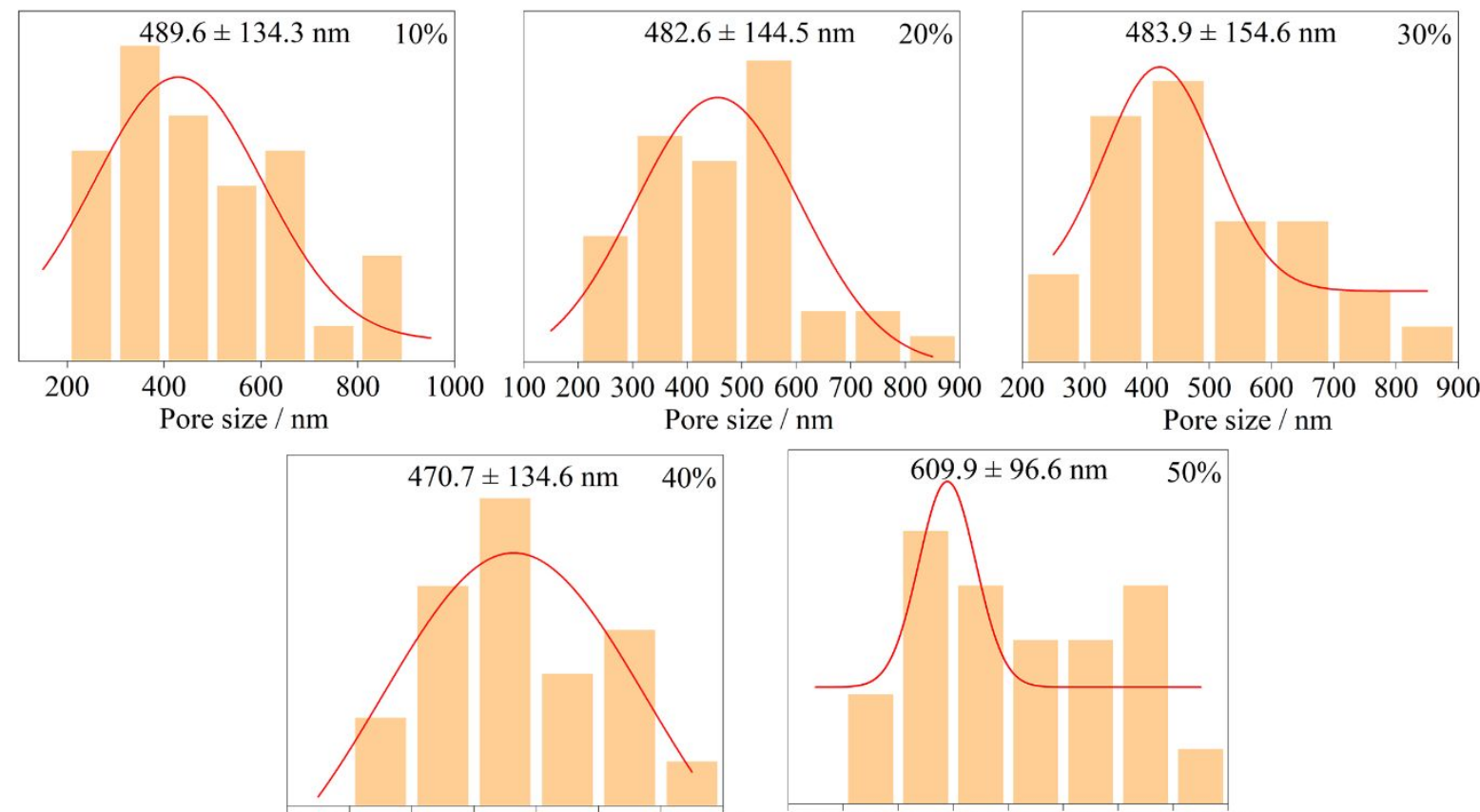

$100200300400500600700 \quad 800 \quad 400450500550600650700750800$

Pore size / nm

Pore size / nm

Figure S1 Pore size distribution of the $10-50 \%$ samples. 


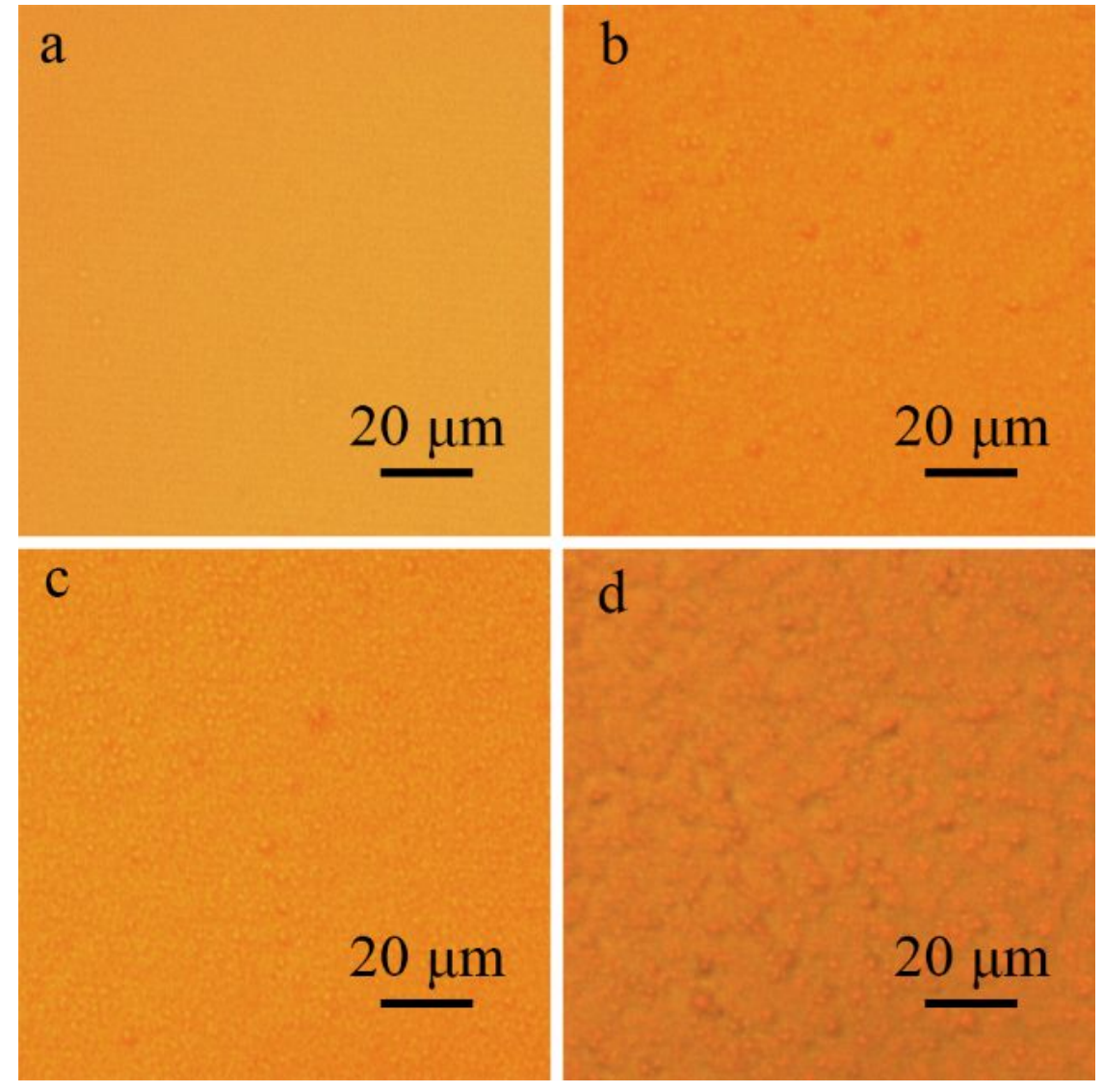

Figure S2 Optical microscopy images of pristine Sylgard 184 (a), 10\% (b), 30\% (c) and 50\% (d) coatings. Spots (pores) are mono-dispersed inside the structure of the $10-50 \%$ sample in contrast to pristine Sylgard 184 coating almost without anything. The density of the spots increases with the increase of the porosity. 


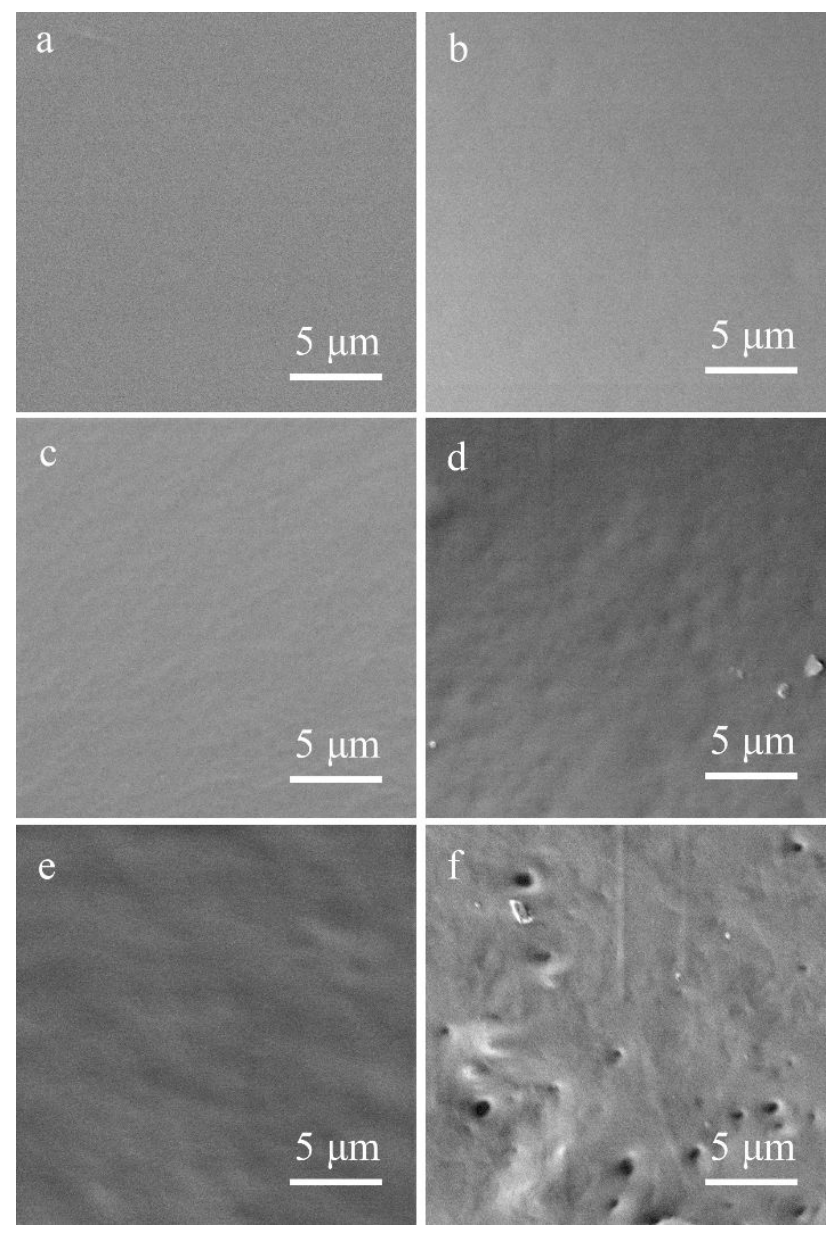

Figure S3 Top-view SEM images of samples' surfaces prepared by spin-coating method with thicknesses of $50 \mu \mathrm{m}$. (a) Pristine Sylgard 184, (b) 10\%, (c) $20 \%$, (d) $30 \%$ (e) $40 \%$ and (f) $50 \%$ samples. The surfaces of $0-40 \%$ samples are intact without pores, while the $50 \%$ sample has pores on its surface. 


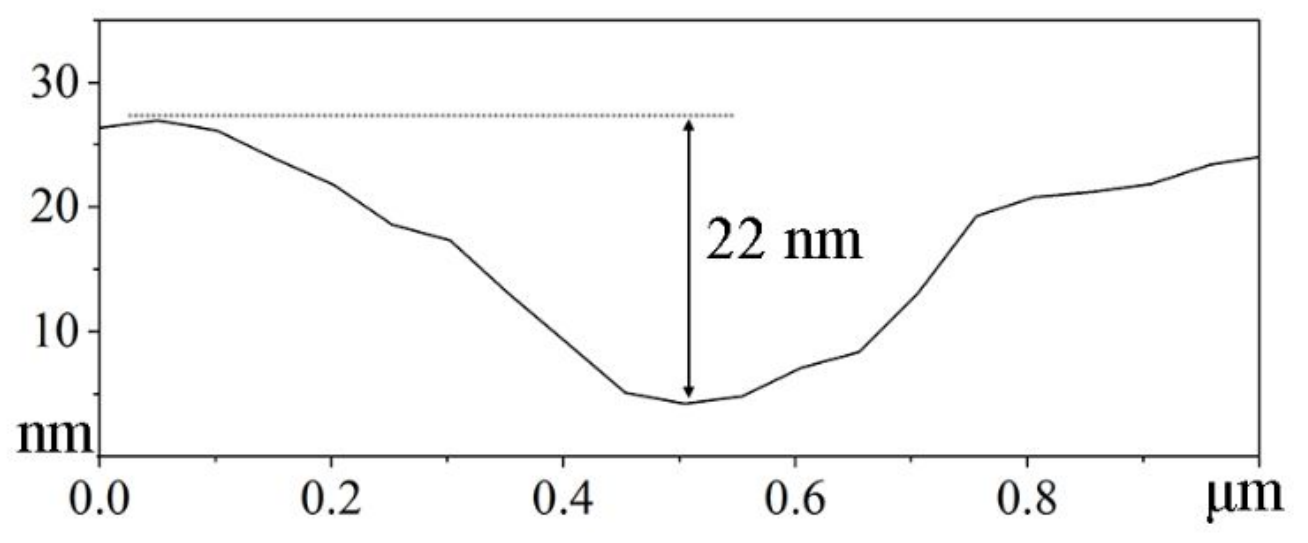

Figure S4 Height profile along the white line in Figure $3 \mathrm{c}$ as an example. The average of the depth of the dents was obtained by the average value of 20 dents. 

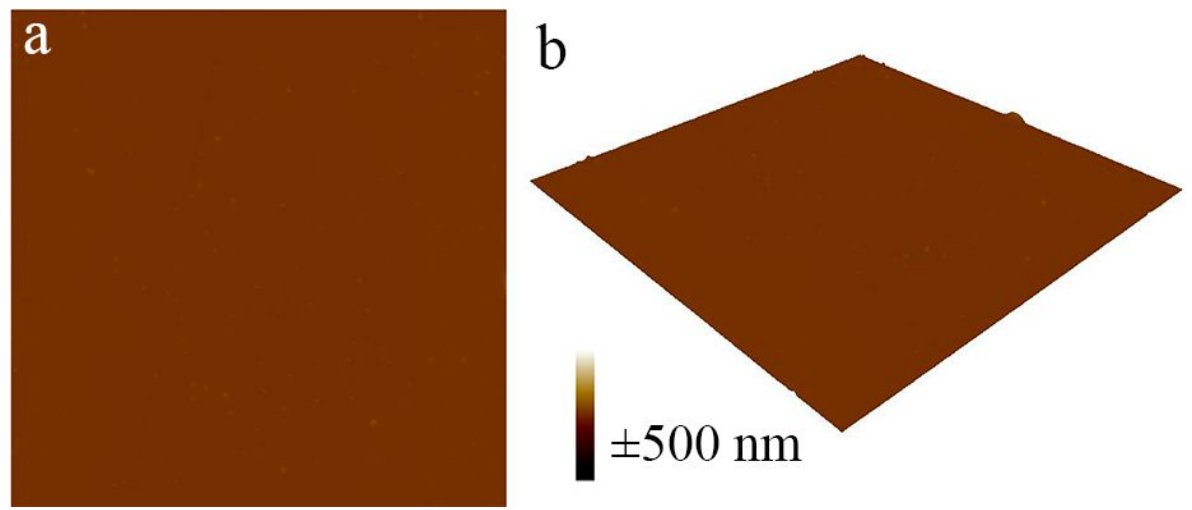

Figure S5 (a) Two- and (b) three-dimensional AFM images of pristine Sylgard 184 coating. 

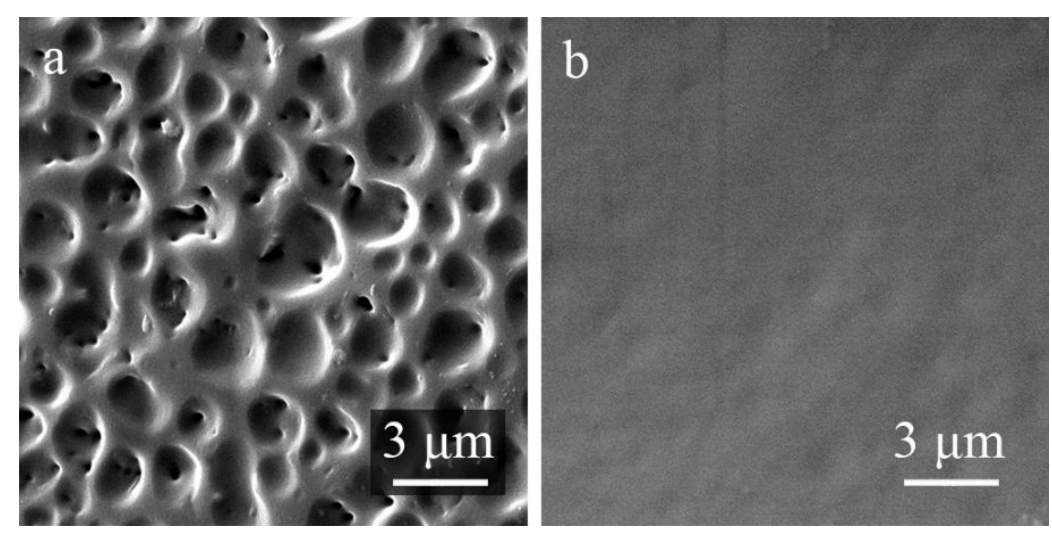

Figure S6 Top-view of SEM images of the (a) porous and (b) smooth surfaces. 


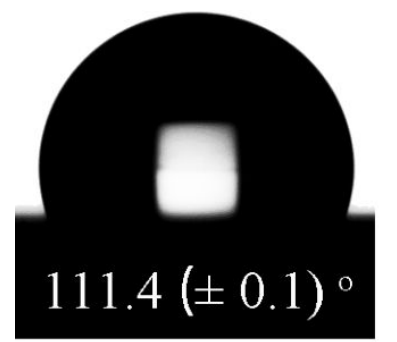

PDMS

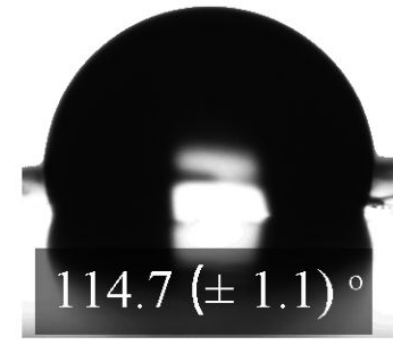

$30 \%$ sample

Figure S7 Water contact angles of pristine PDMS surface (Sylgard 184) and 30\% sample. 

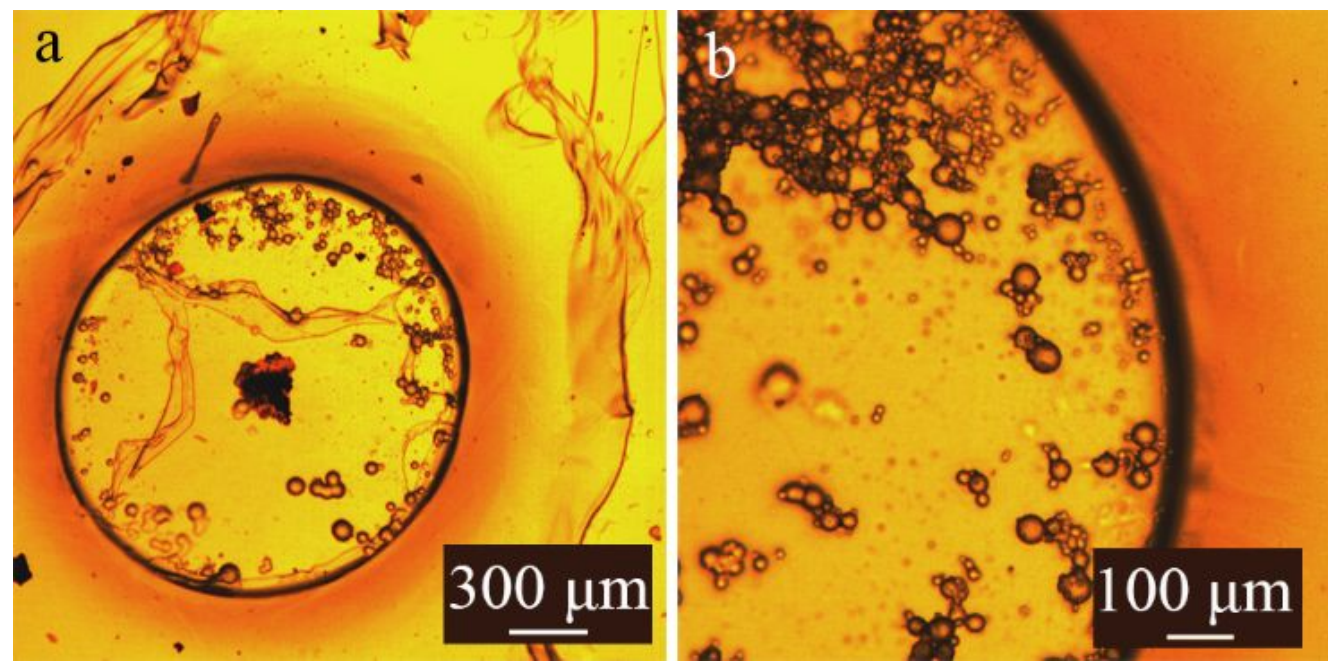

Figure S8 Optical microscopy images of $60 \%$ sample. Macroscopically phase separation between the porogen and Sylgard 184 component, resulting in a fragmentized coating structure. 

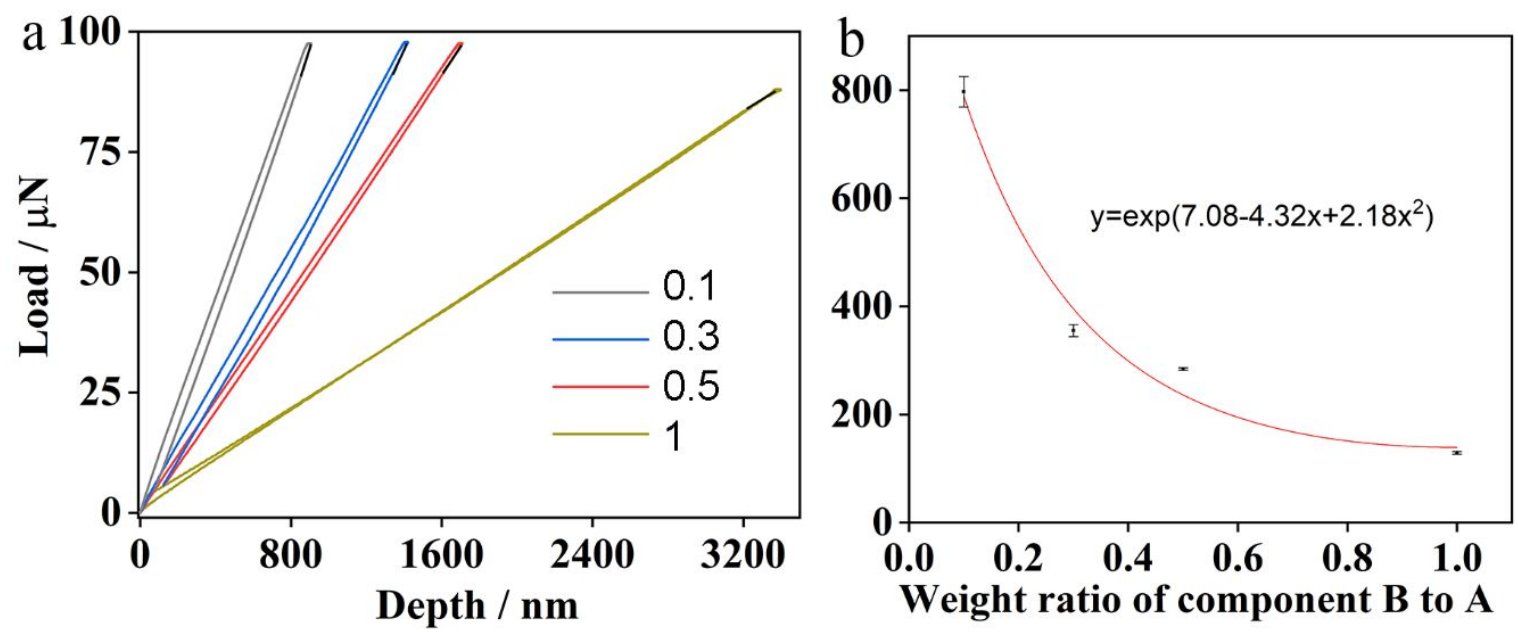

Figure S9 (a) Load-displacement curves of bulk Sylgard 184 PDMS with variable component ratios. (b) Shear modulus of the bulk PDMS as a function of weight ratio of component B to A. 

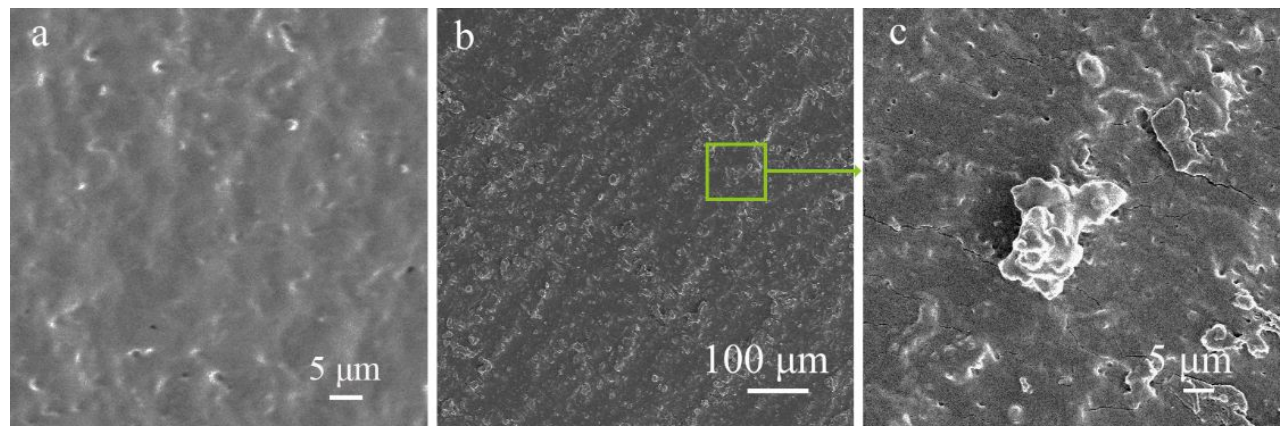

Figure S10 SEM images of the 30\% coating after (a) 5 icing/deicing cyclic test and (b,c) 1000 abrasion cycles. The coating was scuffed by the 400 -grit sandpaper. 


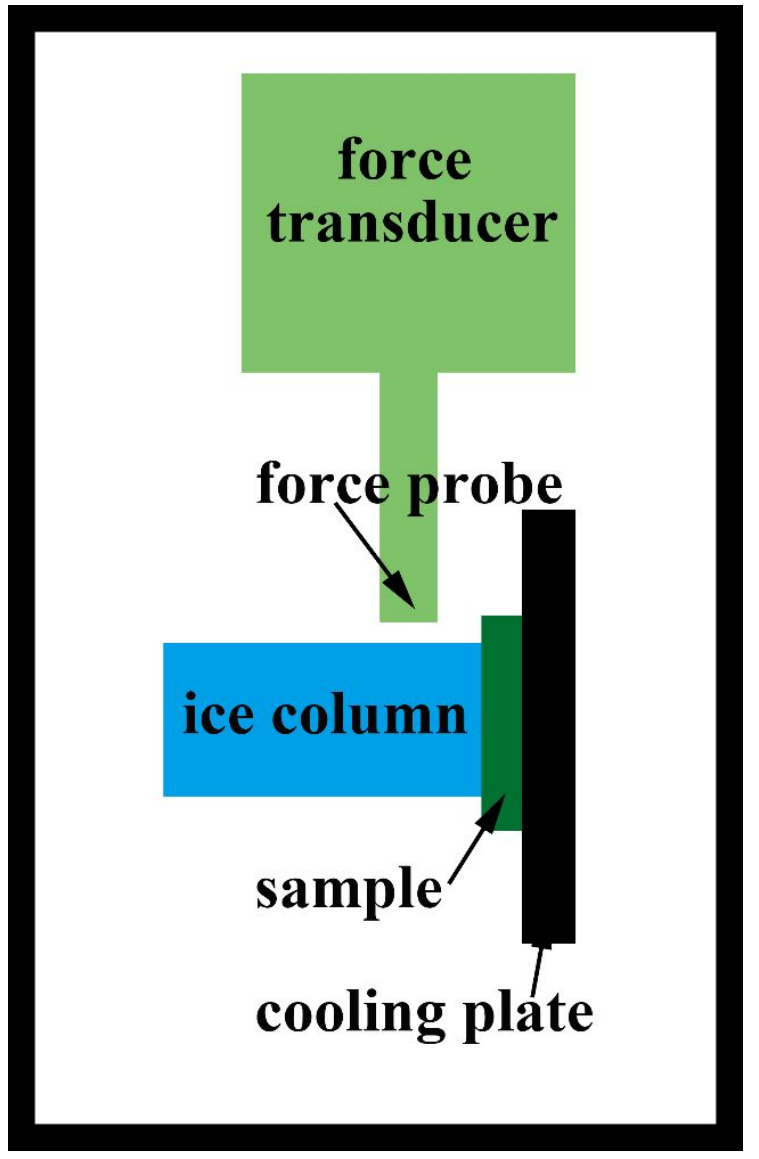

Figure S11 Schematic of the ice adhesion test. A force probe with $5 \mathrm{~mm}$ diameter propelled the tube-encased ice columns under a velocity of $0.01 \mathrm{~mm} \mathrm{~s}^{-1}$, with the probe aligned close to the tested coating surface (less than $2 \mathrm{~mm}$ ) to minimize the torque on the ice cylinder. 


\section{Reference}

(1) Wang, Z.; Volinsky, A. A.; Gallant, N. D. Nanoindentation study of polydimethylsiloxane elastic modulus using berkovich and flat punch tips. Journal of Applied Polymer Science 2015, $132(5), 41384$.

(2) Johnston, I. D.; McCluskey, D. K.; Tan, C. K. L.; Tracey, M. C. Mechanical characterization of bulk Sylgard 184 for microfluidics and microengineering. Journal of Micromechanics and Microengineering 2014, 24 (3), 035017.

(3) Huang, C.; Bian, Z. G.; Fang, C. F.; Zhou, X. L.; Song, J. Z. Experimental and theoretical study on mechanical properties of porous PDMS. Journal of Applied Mechanics 2018, 85 (4), 041009.

(4) Dubbelday, P. S.; Rittenmeyer, K. M. In Shear modulus determination of foamed aluminum and elastomers, IEEE 1985 Ultrasonics Symposium, 16-18 Oct. 1985; 1052-1055. 